termination, although this is not apparent with urinalysis. This discrepancy is difficult to explain.

- The flame photometer gave much better results for potassium than for sodium, which showed large variations in accuracy over long time periods, even with a calibration update. Its usefulness in urinalysis is limited by the restrictions of the calibrating procedure, especially for sodium.

- Urine chloride determination is restricted similarly to that of sodium and potassium.

- Calcium determination is not acceptably precise. This is probably a reagent problem, because some instability of the color reagent was noted.

- The precision of enzyme activity determination was generally good, although measurements are probably being made just as constant temperature is attained. This may be affecting the precision of aminotransferase activity determination.
We thank Dr. J. M. Erikson, Hycel Inc., Mr. D. Giles, Boehringer Corporation (London) Ltd., and Mr. R. D. Jennings, Department of Health and Social Security, for their invaluable help and that of many from their staffs. Also the help and advice from members of many departments of the Clinical Research Centre and Northwick Park Hospital, Harrow, U.K. We are also indebted to Mr. W. I. R. Martin, Ryde, Isle of Wight, U.K. and Mr. J. P. Royston, Division of Computing and Statistics, Clinical Research Centre, for their help with the statistical analysis.

\section{References}

1. Broughton, P. M. G., Gowenlock, A. H., McGormack, J. J., and Neill, D. W., A revised scheme for the evaluation of automatic instruments for use in clinical chemistry. Anal. Clin. Biochem. 11, 207 (1974).

2. Keen, J., and Page, D. J., Estimating variability from the difference between successive readings. Appl. Stat. 2, 13 (1953).

\title{
Free-Cortisol Assay by Immunoextraction: Comparison with an Equilibrium Dialysis Procedure
}

\author{
Aldo Clerico, ${ }^{1}$ Maria Grazia Del Chicca, ${ }^{1}$ Giancarlo Zucchelli, ${ }^{1}$ Carlo Bartolomel, ${ }^{2}$ and Nedo Riccioni ${ }^{2}$
}

We describe a new method for directly determining the apparent free cortisol concentration in plasma samples by use of an antibody-coated test-tube RIA. Buffer-diluted plasma or standard serum is added to antibody-coated test tubes, incubated at $4{ }^{\circ} \mathrm{C}$ for $4 \mathrm{~h}$, and the solutions are aspirated. ${ }^{125}$-labeled cortisol is added to each tube and incubated for $3 \mathrm{~h}$ at $4^{\circ} \mathrm{C}$. Then the insides of the tubes are washed and their radioactivities counted. The standard curve is in terms of free cortisol, the standard serum solutions having been measured with an equilibrium dialysis procedure. Plasma samples $(n=155)$ from normal subjects and from various patients, measured with the new immunoextraction method and the equilibrium dialysis technique, gave results that correlated well $(r=0.847, p$ $<0.001)$. Results by this direct RIA also correlate well with the clinical adrenocortical status of patients for whom data on total plasma cortisol may be misleading. This simple, easy RIA is suited to be the routine method for free cortisol in plasma.

The free (non-protein-bound) fraction of steroid hormones has a major role in determining their biological activity. Therefore, assay of free steroid appears to furnish more-reliable information about the functional status of these hormones, especially in cases where the binding capacity of plasma proteins is altered.

Recently, we described a highly sensitive method for determining the apparent free cortisol concentration (AFCC)

${ }^{1}$ Institute of Clinical Physiology, C.N.R., University of Pisa, Via Savi, 8-56100 Pisa, Italy.

2 City Hospital, Pontedera, Pisa, Italy.

Received Aug. 3, 1981; accepted Feb. 5, 1982. in plasma samples by means of an RIA in which an antiserum is directly coated on the test tube wall (1). The diffusible (free) cortisol in the plasma dialysate after an equilibrium dialysis (ED) is measured directly by this RIA method.

However, ED has the disadvantage of a long incubation interval with respect to other techniques that are available for the measurement of the free hormone fraction, such as gelequilibrium, ultrafiltration, and ultracentrifugation. Additional drawbacks are the volume of plasma necessary for the assay $(1 \mathrm{~mL})$ and the need to determine AFCC by two steps (ED and RIA).

Therefore, we have developed a direct assay of free cortisol in plasma, in which the same antibody-coated-tube RIA is used as for the ED procedure (1). Our procedure is based on the immunoextraction of cortisol in unknown samples, which is measured from a calibration curve prepared by use of human serum standards in which the free hormone content was previously determined by the ED system (1) as the comparison method.

Here we describe the methodological characteristics of this new method and compare results with those by the previously described ED procedure.

\section{Materials and Methods}

AFCC was measured in plasma samples by the RIA plus ED technique as previously described $(1,2)$. The ED was performed with $1 \mathrm{~mL}$ of plasma at $37^{\circ} \mathrm{C}$ for $20-24 \mathrm{~h}$ vs $5 \mathrm{~mL}$ of buffer. The diffusible cortisol in the dialysate was then measured by an RIA method in which an antiserum is directly coated on the test tubes and ${ }^{125}$ I-labeled cortisol is used as the tracer [SPAC Cortisol, kindly supplied by Byk Gulden Italia S.p.A., Cormano (MI), Italy] (1).

This same RIA method was also used for the direct assay 


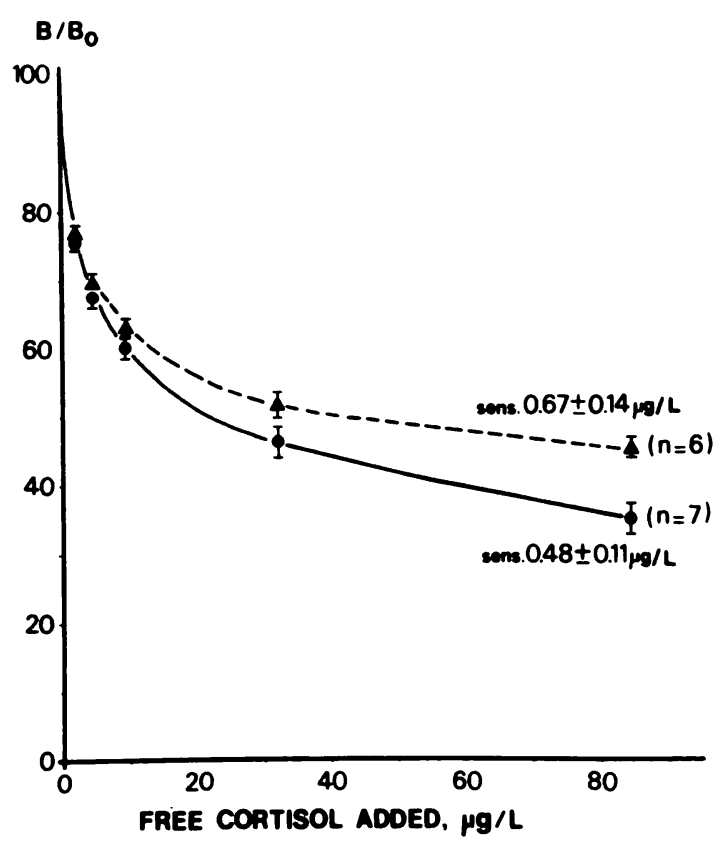

Fig. 1. Standard curves with two different lots of tubes

of AFCC in plasma or serum samples. The assay was performed as follows: add $0.2 \mathrm{~mL}$ of unknown plasma samples or of human serum standard to test tubes and dilute with $0.8 \mathrm{~mL}$ of phosphate buffer ( $150 \mathrm{mmol} / \mathrm{L}, \mathrm{pH} 7.4)$. Incubate the tubes for $4 \mathrm{~h}$ at $4^{\circ} \mathrm{C}$ and discard the solution, then add $1 \mathrm{~mL}$ of 125I-labeled cortisol solution (about $1 \mu \mathrm{Ci}$ used for 50 test tubes; also supplied by Byk Gulden Italia S.p.A.). After a further incubation at $4^{\circ} \mathrm{C}$ for $3 \mathrm{~h}$, discard the solution and wash the insides of the tubes with $1.5 \mathrm{~mL}$ of isotonic saline solution. Discard the wash solution and count the radioactivity in the tubes in a well-type scintillation counter for 1-2 min.

Prepare the standard curve in such a way as to express the standard solutions (normal human sera with standard cortisol of well-known total amount, kindly supplied by Travenol, Clinical Assay, Rome, Italy) in terms of free cortisol content, as independently measured with the ED technique.

We measured 155 plasma samples by the new direct RIA method and by the ED technique. The samples were from seven normal persons; 21 pregnant women; eight patients with cirrhosis of the liver and nine with anorexia nervosa; and from one patient with carcinoma of the prostate, who was undergoing treatment with estrogen, sampled in basal condition at different hours of the day and (or) after stimulation or suppression tests.

The sensitivity, the index of precision, and the sample values were calculated by use of a calculator program previously described (3).

\begin{tabular}{|c|c|c|}
\hline $\begin{array}{l}\text { Total cortbol } \\
\text { concn, } \mu \mathrm{g} / \mathrm{L}\end{array}$ & $\begin{array}{l}\text { Moan (SD) } \\
\text { AFCC, } \mu g / L\end{array}$ & cV, \% \\
\hline 20 & $2.2(0.8)$ & 36.4 \\
\hline 50 & $4.4(1.3)$ & 29.6 \\
\hline 100 & $10.2(1.5)$ & 14.7 \\
\hline 250 & $32.3(1.1)$ & 3.4 \\
\hline 600 & $88.8(13.1)$ & 14.8 \\
\hline
\end{tabular}

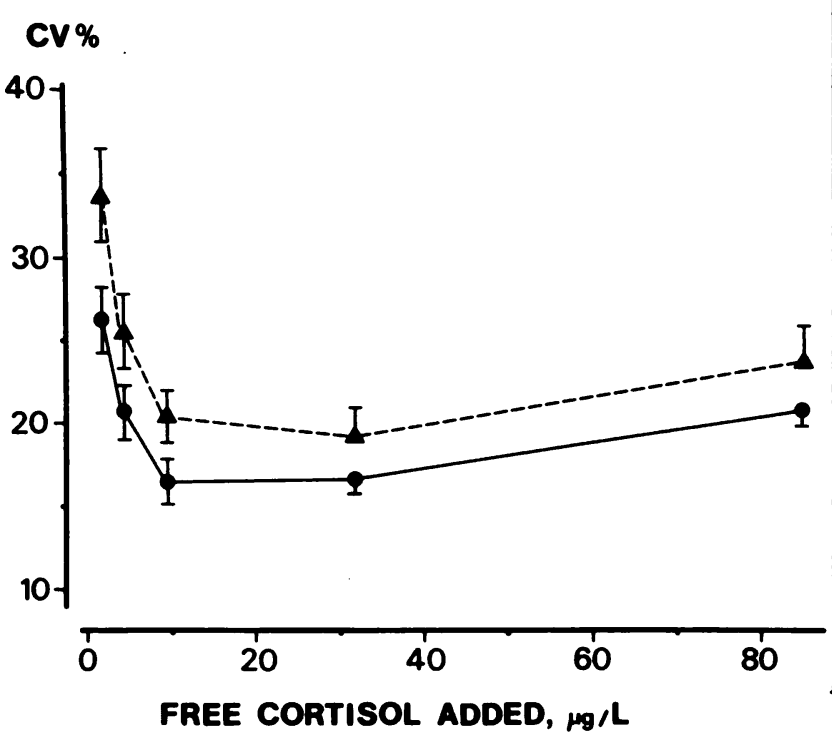

Fig. 2. Mean precision profiles

\section{Results}

Standard curve and sensitivity. A great variation in stan dard curves obtained resulted when different lots of anti body-coated-test tubes were used. The mean standard curves ( \pm SEM) obtained with two different lots of tubes are illus trated in Figure 1, as is the mean sensitivity ( $(\mathrm{SEM})$ of these two curves.

Four lots of standards were used for the free cortisol assay by our direct RIA procedure. Table 1 reports the values obtained on measuring these lots by means of $\mathrm{ED}$ in six different experiments.

Precision. The mean precision profiles of the two standard curves of Figure 1 are shown in Figure 2. As expected, the more sensitive standard curve also shows better precision. The between-assay variability (CV), obtained repeatedly on measuring one plasma pool collected from blood donors in different assays $(n=25)$ during one year, with use of severa lots of antibody-coated-test tubes, was $25.5 \%$ (mean $\pm \mathrm{SD}=$ $11.0 \pm 2.8 \mu \mathrm{g} / \mathrm{L}$ ). For the same pool the between-assay vari ability found by $\mathrm{ED}$ was $20.0 \%(n=16$, mean $\pm \mathrm{SD}=11.0 \pm$ $2.2 \mu \mathrm{g} / \mathrm{L})$.

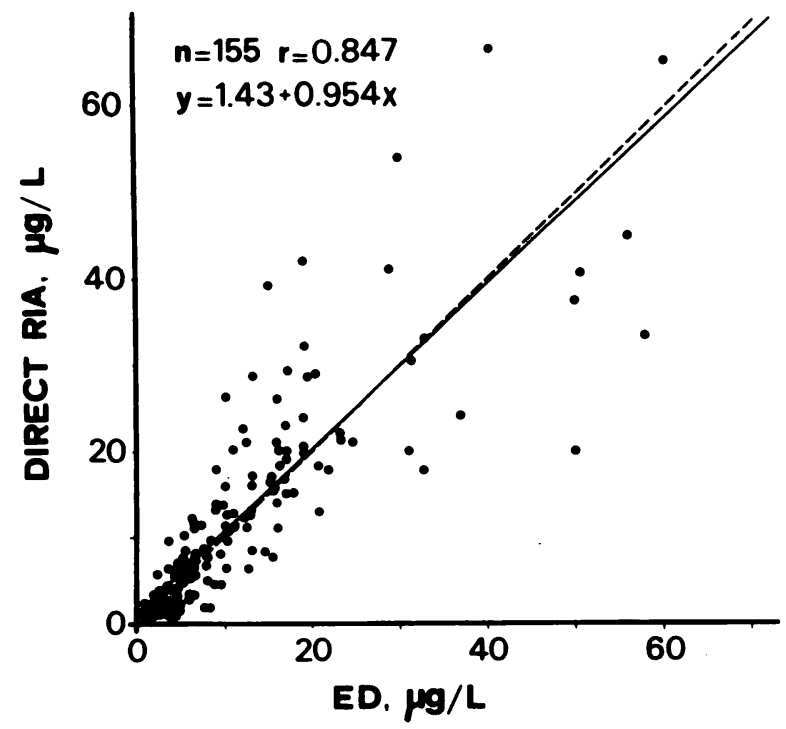

Fig. 3. Correlation between the values by the present method and by the equilibrium dialysis technique ( 1 )

The dashed line is the line of identity; the linear regression between the two series of values is indicated by the continuous line 


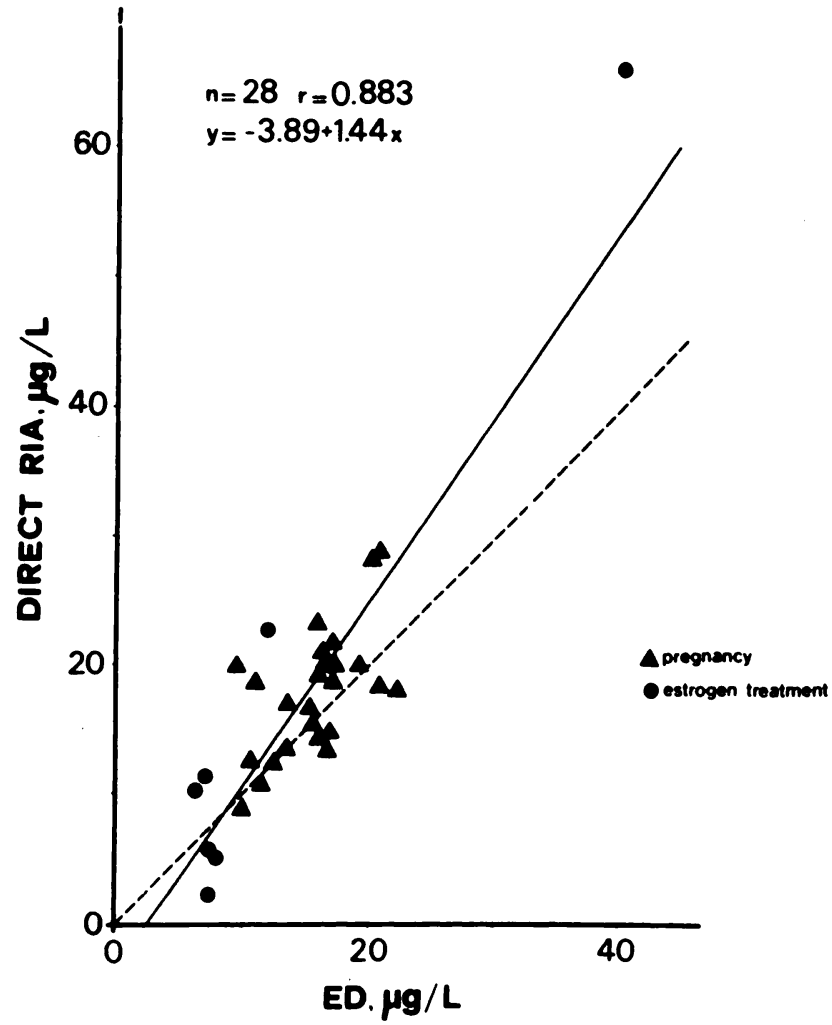

Fig. 4. Correlation between values by two methods, for plasma samples from a patient with carcinoma of the prostate and for pregnant women

Dashed line: represents the line of identity; continuous line, the linear regression

Comparison with ED technique. Values obtained for the 155 plasma samples with the new assay and by the ED technique are reported in Figure 3. A strong correlation was found between results by the two procedures $(p<0.001)$. A similar degree of correlation was found in the case of the samples from the patient with carcinoma of the prostate, who was undergoing treatment with estrogens and who had high values for total cortisol $(208-1109 \mu \mathrm{g} / \mathrm{L})$, and for samples from 21 pregnant women (Figure 4). A similar degree of correlation was also found in the case of all the other groups of subjects studied (normals, anorexia nervosa, liver disease).

\section{Discussion}

This new method showed good sensitivity (Figure 1), permitting determination of AFCC in plasma samples containing very low free cortisol concentrations (normal range 3.5-17.0 $\mu \mathrm{g} / \mathrm{L}$ ). An appropriate choice of antibody-coated tubes results in better sensitivity and adequate precision for the assay (Figures 1 and 2). However, even with no selection of tubes, the precision of the determination is not very different $(26 \%)$ from that found, during a year's experiments, by ED with the same pool (20\%). On the other hand, the new method has some advantages over the ED technique: AFCC can be measured in plasma in about one day, as compared with two days with the ED technique; only $0.2 \mathrm{~mL}$ of plasma is required $(1 \mathrm{~mL}$ with ED); and with the same materials one can determine concentrations of both free and total cortisol.

Although the cortisol immunoextracted by the antiserum-coated-tubes was not the theoretical free-hormone content of the plasma sample, this new method shows a high correlation with AFCC measured with the comparison method (ED system), even in particular physiopathological conditions in which the protein binding of plasma samples was considered to be grossly altered (pregnancy, estrogen treatment, cirrhosis), whereas the total plasma cortisol may give misleading results in such conditions. Therefore, because this method is simple and easy to perform, it could well become a useful addition to routine laboratory tests for adrenocortical function investigation.

\section{References}

1. Del Chicca, M. G., Clerico, A., Ferdeghini, M., et al., Radioimmunoassay of free cortisol with antiserum-coated tubes and ${ }^{125}$ I-labeled cortisol. Clin. Chem. 26, 1231-1232 (1980).

2. Clerico, A., Del Chicca, M. G., Zucchelli, G. C., et al., Radioimmunological determination of free cortisol in an equilibrium dialysis system. J. Nucl. Med. Allied Sci. 22, 5-10 (1978).

3. Pilo, A., and Zucchelli, G. C., Automatic treatment of radioimmunoassay data: An experimental validation of the results. Clin. Chim. Acta 64, 1-9 (1975). 